\title{
Caractérisation du comportement mécanique d'un composite (tissu de verre/résine époxyde) à différentes périodes de vieillissement hygrothermiques
}

\author{
A. NACERI ${ }^{1, *}$ et A. VAUTRIN ${ }^{2}$ \\ 'Laboratoire de matériaux, Faculté des sciences de l'ingénieur, Université de M'sila, \\ B.P 166, Ichbilia, M'sila 28000, Algérie \\ ${ }^{2}$ Laboratoire de mécanique et matériaux, Ecole des mines de Saint-Etienne, 158 Cours \\ Fauriel, 42023 Saint-Etienne, France
}

(Reçu le 19 Octobre 2005, accepté le 12 Mars 2006)

*Correspondance,courriel : abdelghani_naceri@yahoo.fr

\section{Résumé}

Cet article présente l'analyse du comportement mécanique d'un stratifié composite constitué de 12 plis de tissus de fibres de verre/résine époxyde conditionné en milieu humide à différents taux d'humidité relative 0,60 et $96 \%$ à $60^{\circ} \mathrm{C}$.

L'analyse des résultats expérimentaux obtenus du vieillissement hygrothermique sur la réponse mécanique a permis de montrer que l'influence de la concentration en eau sur les caractéristiques ultimes devient significative et importante pour le composite conditionné à $96 \%$ d'humidité relative aux époques I et II (plateau de saturation et vieillissement).

Mołs-clés : Vieillissement hygrothermique, comportement mécanique, matériaux composites.

\section{Abstract}

Characterization of the mechanical behaviour of composite (woven fabric glass/epoxy resin) at different times during hygrothermal ageing

This paper considers the analysis of the mechanical behaviour of a laminate constituted of 12 layers of glass fiber fabric/epoxy resin conditioned at different relative humidities of 0,60 and $96 \%$ at $60^{\circ} \mathrm{C}$.

The analysing of the experimental results obtained of hygrothermal ageing on the mechanical response has permited to show that the influence of the moisture concentration on the ultimate mechanical properties becomes significant and important 
for the composite conditioned at relative humidity of $96 \%$ to the periods I and II /state of saturation and ageing).

Keywords : Hygrothermal ageing, mechanical behaviour, composite materials.

\section{Introduction}

L'expérience de ces dernières années souligne l'importance des effets de l'environnement (humidité et température) sur les propriétés mécaniques des plastiques renforcés [1] et la stabilité de leurs performances à long terme (durabilité).

La présence d'eau au sein des matériaux composites (fibres/polymère) provoque une détérioration des propriétés mécaniques [2].

On sait qu'au cours d'un vieillissement hygrothermique, la présence de molécules d'eau dans un composite à matrice polymère peut notamment dégrader les liaisons à l'interface renfort-matrice et entraîner ainsi une dégradation irréversible des propriétés du composite [3].

Ce sont surtout les applications aéronautiques qui ont initié l'étude des problèmes liés à l'environnement [3-4]. L'absorption d'eau [5] par un composite à matrice organique dépend de plusieurs paramètres tels que :

- la nature des fibres et de la résine,

- la disposition des fibres par rapport à la direction de diffusion,

- l'écart de concentration en eau entre le composite et le milieu environnant.

L'objectif principal de cette étude expérimentale consiste à étudier l'influence de l'environnement hygrothermique sur le comportement mécanique d'un stratifié composite constitué de 12 plis de tissus de verre $E$ et d'une résine époxyde saturé à différents taux d'humidité relative 0,60 et $96 \%$ RH à la température constante de $60^{\circ} \mathrm{C}$. Dans cette étude les éprouvettes sont sollicitées jusqu'à rupture en traction uniaxiale dans les deux directions, chaîne et trame à vitesse de déplacement imposée constante $(\mathrm{V}=0,011 \mathrm{~mm} / \mathrm{s})$.

\section{Matériau, conditionnement et essai mécanique}

L'étude porte sur un composite constitué de 12 couches (937/EP/ES67) de tissus à armure taffetas ou toile (le fil de chaîne et de trame s'entrecroisent alternativement) de fibres de verre $\mathrm{E}$ noyé dans une résine époxyde holt melt. Ce matériau a été réalisé par la société Hexcel Génin. II est délivré sous forme de plaques planes de dimensions moyennes: 300 (sens trame) $\times 300$ (sens chaîne) $\times 3,2 \mathrm{~mm}^{3}$. La fraction volumique des fibres a été déterminée selon la méthode de calcination et trouvée égale à $V_{f}=55 \%$. 
La technique de mesure utilisée pour la détermination de la masse volumique du composite tissu est la méthode picnométrique $\left(1,94 \mathrm{~g} / \mathrm{cm}^{3}\right)$. Sa composition pondérale est égale à $50 \%$ dans chaque direction de renforcement fibreux (sens chaîne et trame).

La détermination de la cinétique d'absorption d'eau du matériau étudié a été effectuée par une mesure de reprise de poids en fonction du temps dans le but de déterminer les deux paramètres caractéristiques de la loi de diffusion de FICK qui admet la réversibilité du phénomène :

- le coefficient de diffusion $D$ en $\mathrm{cm}^{2} / \mathrm{s}$, et

- la quantité maximale d'eau retenue à la saturation $M_{m}$ en $\%$.

La loi de diffusion de FICK admet que le moteur de diffusion n'est autre que le gradient de concentration en eau. L'équation de bilan de matière s'écrit [6-7] :

$$
\frac{\partial C}{\partial t}=\frac{\partial}{\partial x_{i}}\left(D_{i j} \frac{\partial C}{\partial x_{j}}\right)
$$

Il s'agit d'une équation différentielle aux dérivées partielles, où la concentration en eau C dépend des variables spatiales $x_{i}$ et du temps $t$.

où :

$D_{i j}:$ diffusivité du milieu,

$C$ : concentration en eau.

En ce qui concerne la diffusivité $D$, on la suppose dans ce modèle indépendante de l'humidité relative et seulement fonction de la température suivant une loi de type Arrhénius :

$$
D=D_{0} \exp \left(-\frac{E_{d}}{R T}\right)
$$

avec,

$D$ : coefficient de diffusion ou diffusivité,

$R$ : constante des gaz parfaits,

$E_{d}$ : énergie d'activation du processus de diffusion,

$\mathrm{T}$ : température absolue.

Par contre la quantité maximale d'eau retenue $M_{\mathrm{m}}$ à la saturation apparaît comme indépendante de la température et seulement fonction de l'humidité relative suivant une loi puissance :

$$
M_{m}=a(R H)^{b}
$$

où ,

$\mathrm{RH}$ : humidité relative du milieu en \%,

$a$ et $\mathbf{b}$ : constantes du milieu. 
Le coefficient de diffusion $D$ et la quantité maximale d'eau retenue à la saturation $M_{m}$ sont deux paramètres matériels fondamentaux qui caractérisent l'aptitude du matériau à absorber l'eau dans le cadre de la théorie élémentaire de Fick.

Le choix des conditions d'environnement à température et aux taux d'humidité constants est essentiellement justifié par les deux considérations suivantes:

- au delà de $60^{\circ} \mathrm{C}$, les échantillons pouvaient être endommagés,

- la température choisie de $60^{\circ} \mathrm{C}$ est largement suffisante pour accélérer la reprise en eau et obtenir un palier de saturation en un temps raisonnable.

La cinétique d'absorption d'eau a été suivie par la méthode gravimétrique (reprise de poids mesurée à l'aide d'une balance sartorius 2842 avec une étendue de pesée de $160 \mathrm{~g}$ et une précision de $0,1 \mathrm{mg}$ ) afin de contrôler le gain en poids du matériau conditionné en fonction de la durée de conditionnement.

Le calcul du coefficient de diffusion $D$ et l'estimation de la quantité maximale d'eau retenue à la saturation $M_{m}$ du composite sont effectués à partir de la courbe maîtresse présentant l'évolution de la quantité d'eau absorbée $M$ en fonction de la racine carrée du temps $(\sqrt{t})$.

L'essai mécanique consiste à soumettre les échantillons à une rampe monotone en traction uniaxiale et analyser l'effet du conditionnement en humidité sur leur réponse mécanique. Le choix de l'essai de traction uniaxiale comme essai de caractérisation mécanique est justifié par le fait qu'il reste facile à mettre en œuvre pour les matériaux composites. L'essai est mené sur des éprouvettes normalisées de formes parallélépipédiques de dimensions moyennes $200 \times 30 \times 3,2 \mathrm{~mm}^{3}$ selon les recommandations de la norme ASTM D3039.

Les échantillons équipés de jauges de déformation et de talons ont été soumis à un essai de traction unaxiale en rampe monotone sur une machine de traction servohydraulique de type MST 810 (équipée de mors auto-serrants), contrôlé en vitesse de déplacement imposé constante $(V=0,66 \mathrm{~mm} / \mathrm{min})$.

\section{Résultats et discussion}

\section{3-1. Environnement hygrothermique}

La courbe de la cinétique d'absorption d'eau (reprise en eau) des éprouvettes conditionnées en milieu vapeur (figure $I$ ) précise le détail des deux lots d'éprouvettes à 60 et $96 \%$ RH à $60^{\circ} \mathrm{C}$ à deux époques différentes (l'époque I qui suppose que le plateau de saturation est atteint et l'époque II qui correspond à l'étude du phénomène de vieillissement après la saturation) qui ont été testées en environnement contrôlé. 


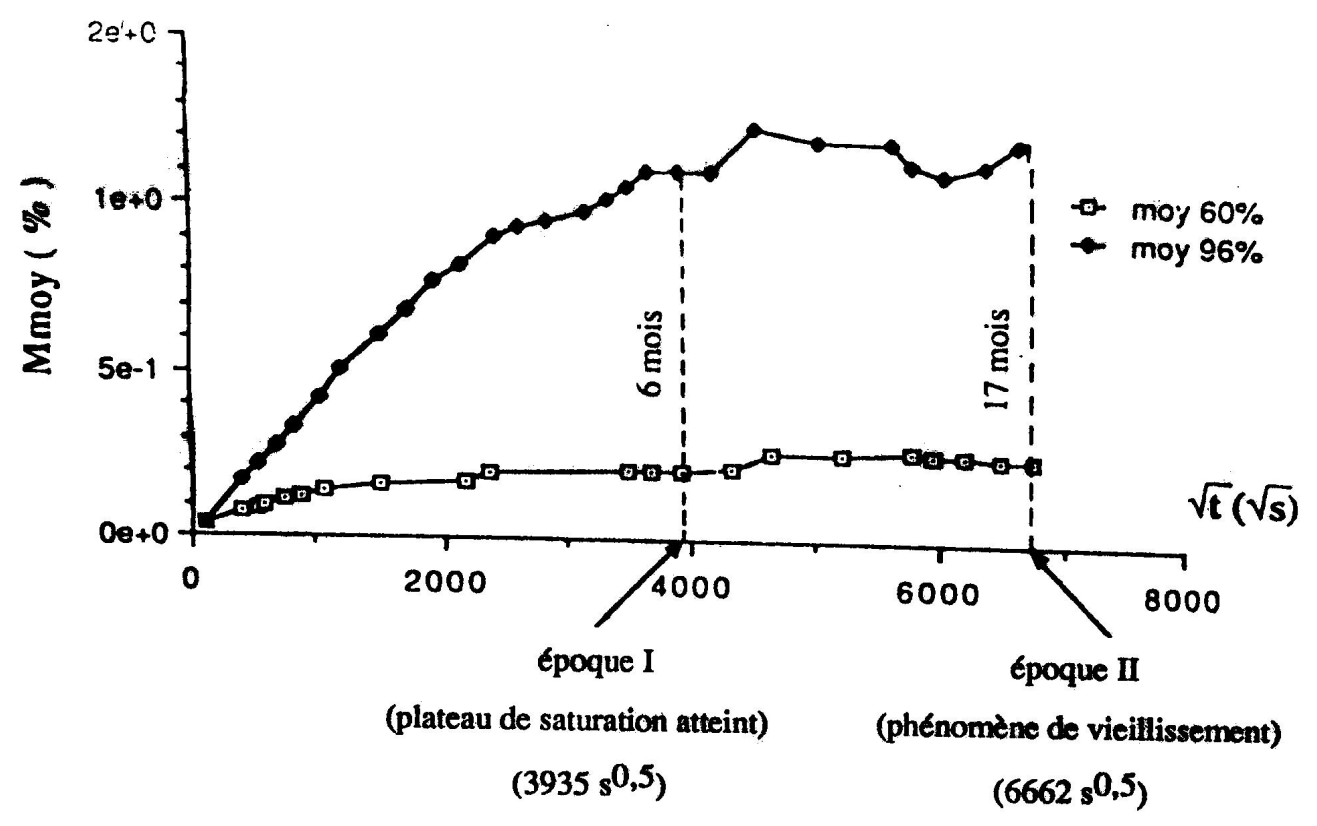

Figure 1 : Courbe de la cinétique d'absorption d'eau du matériau et définition des époques I et I/ d'essais

Le Tableau 1 présente les valeurs des paramètres caractéristiques de la cinétique d'absorption d'eau (la quantité maximale d'eau retenue à la saturation $M_{m}$ et le coefficient de diffusion D) obtenues par l'essai de conditionnement hygrothermique réalisé au laboratoire.

Tableau 1 : Résultats expérimentaux d'absorption d'eau (Mm et D) du composite époxyde/verre E à différents taux d'humidité relative à $60^{\circ} \mathrm{C}$

\begin{tabular}{|c|c|c|}
\hline $\begin{array}{c}\text { Humidité relative } \\
\text { (RH) en } \%\end{array}$ & $\begin{array}{c}M_{\mathrm{m}} \text { en } \\
\%\end{array}$ & $\begin{array}{c}\mathrm{D} \text { en } \\
\mathrm{cm}^{2} / \mathrm{s}\left(\times 10^{-8}\right)\end{array}$ \\
\hline 60 & 0,170 & 1,200 \\
96 & 1,120 & 0,210 \\
\hline
\end{tabular}

Celui-ci confirme clairement les remarques principales observées :

- le coefficient de diffusion $D$ dépend non seulement de la température mais aussi de I'humidité relative, et 
- la quantité maximale d'eau retenue $M_{m}$ à la saturation dépend fortement du taux d'humidité relative.

Les résultats de la cinétique d'absorption d'eau (Tableau 2) obtenues par Loos et Springer [8] du composite polyester/verre E (SMC-30EA) vont dans le même sens que nos valeurs expérimentales d'absorption d'eau $\left(M_{m}\right.$ et $\left.D\right)$. Cette comparaison nous permet de dire que les deux paramètres principaux de diffusion $\left(M_{m}\right.$ et $\left.D\right)$ dépendent non seulement de la température et du taux d'humidité relative mais aussi très fortement de la nature du matériau (type de résine, fibres, etc.).

Tableau 2 : Paramètres caractéristiques de la cinétique d'absorption d'eau du composite polyester/verre E à différents taux d'humidité relative à $65^{\circ} \mathrm{C}[6]$

\begin{tabular}{|c|c|c|}
\hline $\begin{array}{c}\text { Humidité relative } \\
\text { (RH) en } \%\end{array}$ & $\begin{array}{c}\mathrm{M}_{\mathrm{m}} \text { en } \\
\%\end{array}$ & $\begin{array}{c}\mathrm{D} \text { en } \\
\mathrm{cm}^{2} / \mathrm{s}\left(10^{-8}\right)\end{array}$ \\
\hline 60 & 0,45 & 23,0 \\
100 & 2,75 & 3,80 \\
\hline
\end{tabular}

\section{3-2. Réponse mécanique}

Le Tableau 3 présente les résultats expérimentaux obtenus en environnement contrôlé à différents taux d'humidité relative $(0,60$ et $96 \% \mathrm{RH})$ à $60^{\circ} \mathrm{C}$ de l'essai de traction uniaxiale réalisé dans le sens chaîne et trame.

Les valeurs obtenues des modules tangents axiaux $\left(E_{t}\right)$ pour les trois conditionnements hygrothermiques effectués sont presque identiques (influence très faible de l'humidité de l'ordre de $0,4 \%$ pour $60 \%$ RH à $3 \%$ pour $96 \%$ RH pendant l'époque I et de $2 \%$ pour $60 \%$ RH à $4 \%$ pour $96 \%$ RH pendant l'époque II).

Par contre on remarque une diminution significative et marquante de l'ordre de $45 \%$ en moyenne de la contrainte axiale et de la déformation longitudinale de rupture ( $\sigma_{\text {rup }}$ et $\varepsilon_{\text {rup }}$ ) des échantillons conditionnés à $96 \%$ d'humidité relative pendant l'époque I ef II (plateau de saturation et vieillissement). 
Tableau 3 : Résultats des essais mécaniques

\begin{tabular}{|c|c|c|c|c|}
\hline $\begin{array}{c}\text { RH (\%) } \\
\text { à } 60^{\circ} \mathbf{C}\end{array}$ & $\begin{array}{c}\text { Condit- } \\
\text { hygroth }\end{array}$ & $\begin{array}{c}\mathbf{E}_{\mathbf{t}} \\
(\mathbf{G P a})\end{array}$ & $\begin{array}{c}\sigma_{\text {rup }} \\
(\mathbf{M P a})\end{array}$ & $\begin{array}{c}\boldsymbol{\varepsilon}_{\text {rup }} \\
(\%)\end{array}$ \\
\hline $\begin{array}{c}\text { Chaîne } \\
\mathbf{0}\end{array}$ & Sec & 27,82 & 549 & 2,18 \\
\hline $\mathbf{S e c}$ & Epoq I & 27,55 & 520 & 2,47 \\
\hline $\begin{array}{c}\text { Chaîne } \\
\text { Humide }\end{array}$ & Epoq II & 27,35 & 500 & 2,19 \\
\hline $\begin{array}{c}\text { Chaîne } \\
\mathbf{9 6} \\
\text { Humide }\end{array}$ & Epoq I & 27,01 & 313 & 1,31 \\
\hline $\begin{array}{c}\text { Trame } \\
\mathbf{0}\end{array}$ & Epoq II & 26,13 & 310 & 1,26 \\
\hline Sec & Sec & 27,37 & 513 & 2,52 \\
\hline $\begin{array}{c}\text { Trame } \\
\mathbf{6 0} \\
\text { Humide }\end{array}$ & Epoq I & 27,23 & 476 & 2,48 \\
\hline $\begin{array}{c}\text { Trame } \\
\mathbf{9 6} \\
\text { Humide }\end{array}$ & Epoq II & 27,05 & 437 & 2,15 \\
\hline Epoq II & 26,42 & 293 & 1,34 \\
\hline
\end{tabular}

Les évolutions du module tangent axial en fonction de l'allongement longitudinal (Figure 2) pour les trois conditionnements hygrothermiques (sec, deux époques I et II) effectués sont globalement analogues pour une déformation axiale variant de 0 à $1 \%$ (le module tangent axial passe de $27 \mathrm{GPa}$ à $19 \mathrm{GPa}$, soit $30 \%$ de diminution).

On observe également une évolution similaire fortement décroissante (fonction linéaire décroissante) du module tangent axial en fonction de la déformation longitudinale (phénomène lié à l'endommagement des échantillons).

L'équation représentant l'évolution du module tangent axial en fonction de la déformation longitudinale est :

$$
E=E_{0}\left(1-\frac{\boldsymbol{\varepsilon}}{\boldsymbol{\varepsilon}_{0}}\right)=E_{0}(1-a . \boldsymbol{\varepsilon})
$$

où :

$E_{0}:$ module initial tangent axial en $\mathrm{GPa}$, 
$\boldsymbol{\varepsilon}_{0}$ : déformation longitudinale caractéristique en $\%$,

$a$ : coefficient angulaire homogène à $\mathrm{GPa} \%^{-1}$ si $\boldsymbol{E}$ est exprimée en $\%$.
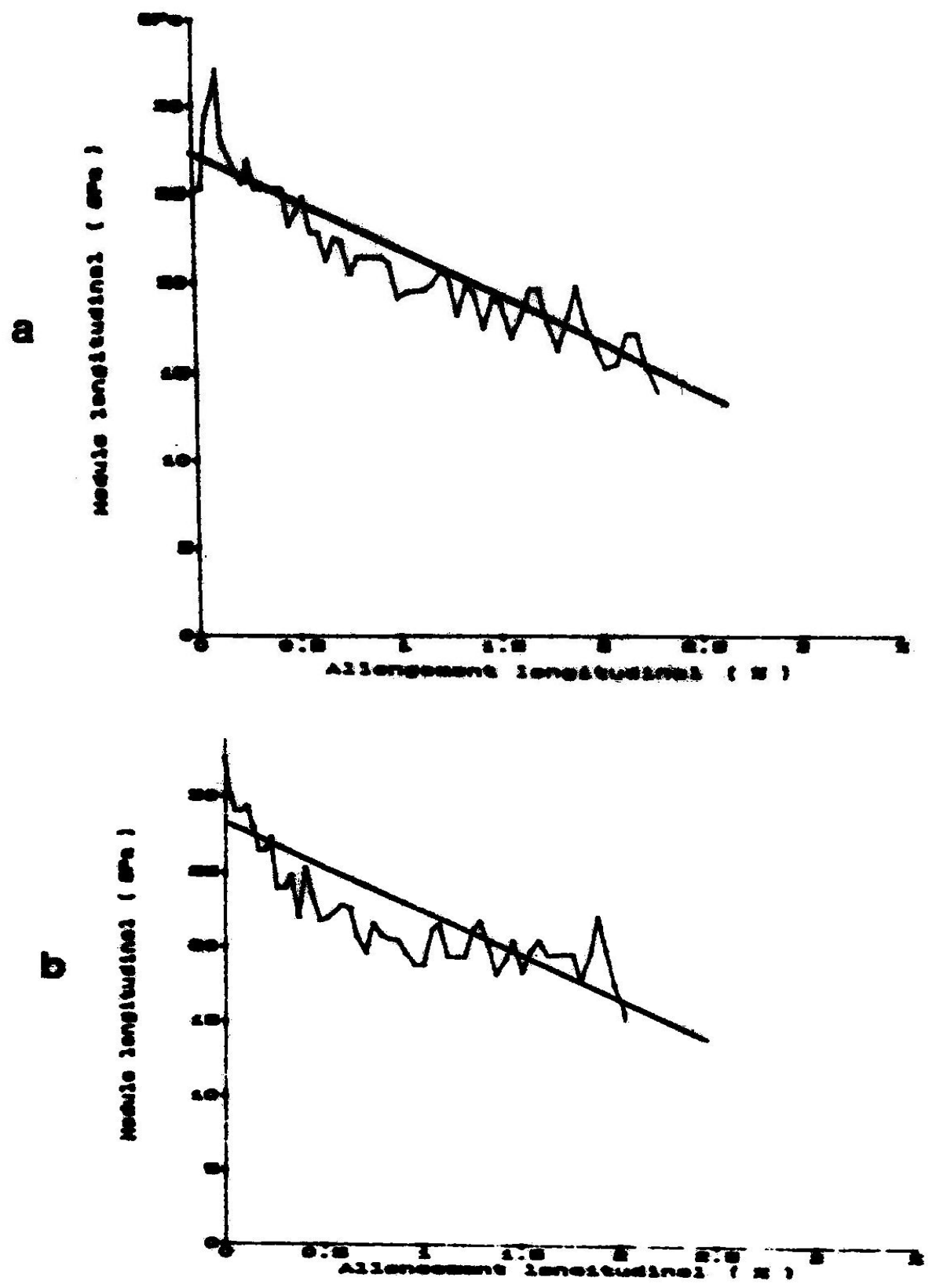

Figure 2 : Evolutions du module tangent axial en fonction de l'allongement longitudinal
a) sens chaîne,
b) sens trame.

A. Naceri et A. Vautrin 
La Figure 3 présente les variations de la contrainte (résistance de traction) axiale de rupture en fonction de la déformation longitudinale de rupture pour le conditionnement hygrothermique (sec, aux époques I et II) réalisé dans le sens chaîne et trame.

On constate donc une décroissance significative de la contrainte axiale de rupture et de l'allongement longitudinal de rupture pour les quantités d'eau absorbées importantes (à $96 \%$ d'humidité relative aux époques I et II du matériau testé). Ceci peut être dô à la fragilisation du composite en présence des molécules d'eau (dégradation des liaisons à l'interface renfort-matrice).

L'équation de la droite (Figure 3) obtenue est la suivante :

$$
\sigma_{r u p}=\sigma_{0}+a \cdot \varepsilon_{r u p}
$$

oÙ :

$\sigma_{0}$ : contrainte caractéristique en $M P a$,

$a$ : paramètre caractéristique (pente de la droite en échelle linéaire).

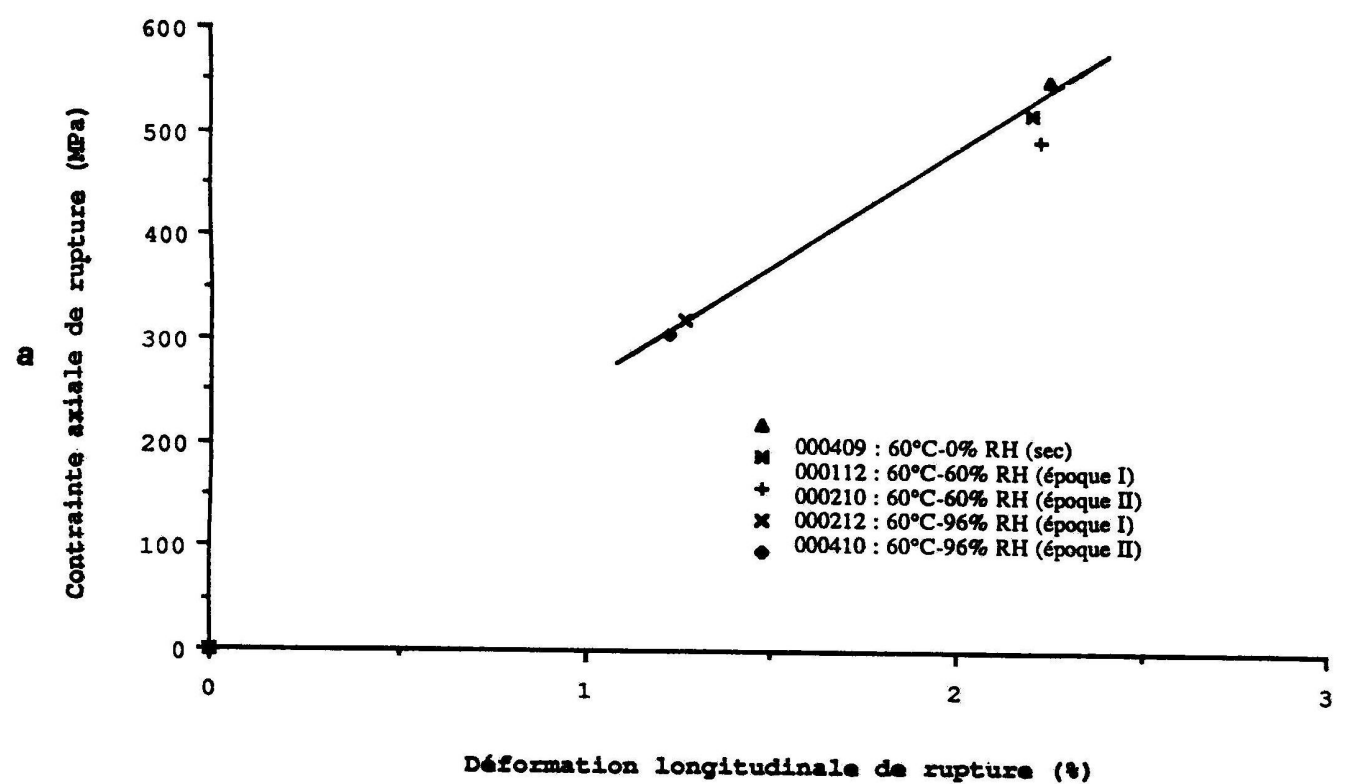

A. Naceri et A. Vautrin 


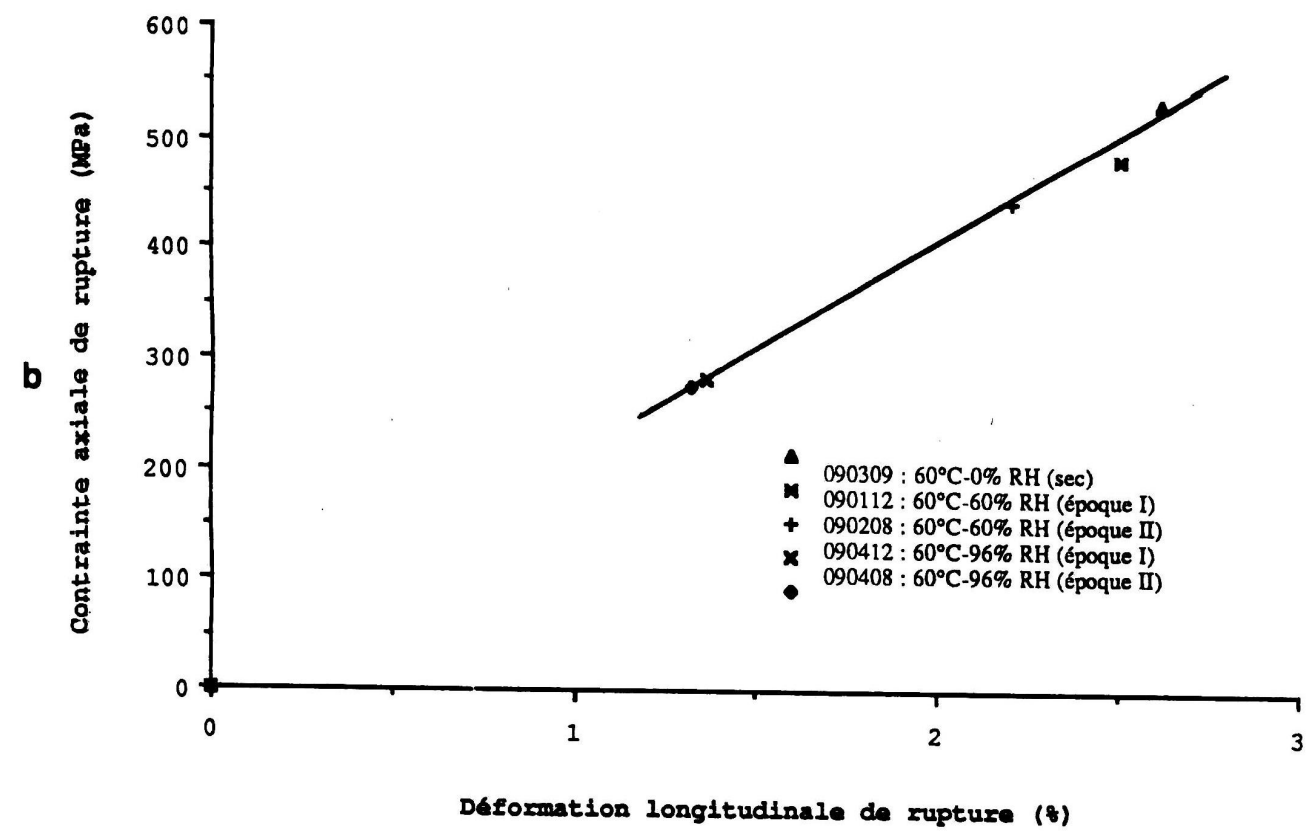

Figure 3 : Variations de la contrainte axiale de rupture en fonction de la déformation longitudinale de rupture
a) sens chaîne,
b) sens trame.

\section{Conclusion}

Bien qu'il y ait une dispersion non négligeable des résultats expérimentaux obtenus, on peut relever que l'absorption d'eau se traduit par une diminution des caractéristiques mécaniques ultimes, cette chute est importante pour les échantillons conditionnés à $60^{\circ} \mathrm{C}-96 \%$ d'humidité relative.

Une reprise en eau entraîne nettement une réduction de la résistance, les échantillons secs étant toujours les plus résistants et les échantillons conditionnés à $96 \%$ RH étant toujours les plus fragiles. 


\section{Références}

[1]- F. FLLYIN and C. ROHRBACHER, "The influence of aqueous environment, temperature and cyclic loading on glass-fiber/epoxy composite laminates", Journal of Reinforced Plastics and Composites, (2003) 615-635

[2] - Y. SURREL and A. VAUTRIN, "On a modeling of the plastic response of FRP under monotonic loading", Journal of Composite Materials, (1999) 232-250

[3] - P. VADDADI, T. NAKAMURA and R.P. SINGH, "Inverse analysis for transient moisture diffusion through fiber-reinforced composites", Acta Materialia, (2003) $177-193$

[4] - S. SRIRAHI, A. REVATHI and R.M.V.C.K. RAO, "Hygrothermal effects on Rt-Cured glass-epoxy composites in immersion environments. Part A: Moisture absorption characteristics", Journal of Reinforced Plastics and Composites, (2002) 983-991

[5] - I. VERPOEST and G.S. SPRINGER, "Moisture absorption characteristics of aramid epoxy composites", Journal of Reinforced Plastics and Composites, (1998) 2-32

[6] - J. ZHOU and Y. ZHONG, "Effects of hygrothermal cycling on properties og glass_vinyl ester composite", Journal of Reinforced Plastics and Composites, (2004) 483-490

[7] - Y. HSIEN-LIANG and Y. HSIEN-YANG, "The effect of lamina material properties on hygrothermal expansion coefficients of angle-play laminatres", Journal of Reinforced Plastics and Composites, (2004) 1673-1681

[8] - A.C. LOOS and G.S. SPRINGER, "Moisture absorption of polyester glass composites", Composite Materials, (1988) 51-62 